\title{
Factors Influencing Nursing Home Accreditation-Quality Ratings in Korea
}

\author{
Shin, Juh Hyun ${ }^{1} \cdot$ Shin, In-Soo ${ }^{2} \cdot$ Lee, Ji Yeon ${ }^{3}$ \\ ${ }^{1}$ Associate Professor, College of Nursing, Ewha Womans University, Seoul \\ ${ }^{2}$ Associate Professor, Department of Transdisciplinary Security, Dongkuk University, Seoul \\ ${ }^{3}$ Graduate Student, College of Nursing, Ewha Womans University, Seoul, Korea
}

\begin{abstract}
Purpose: The purpose of this study is to examine the relationship between the level of competition among nursing homes (NHs) and their geographical locations with government $\mathrm{NH}$ accreditation-quality ratings in Korea. We also examined the possible mediating effects of nurse turnover and $\mathrm{NH}$ residents' hospitalization on the relationship. Methods: We used a cross-sectional design in this study. Data accrued using a disproportionately stratified cluster-sampling method in Korea. Independent variables included location and competition among $\mathrm{NHs}$, as measured by the Herfindahl-Hirschman Index. The mediating variables included registered nurse (RN) turnover and hospital admissions from $\mathrm{NHs}$. The dependent variable was the $\mathrm{NH}$ accreditation grade, openly published by the Korean National Health Insurance Service. Results: The high turnover of professional RNs results in deteriorated government nursing-home-quality ratings and increased hospital admissions relating to better nursing-home-quality ratings. RN turnover was statistically significant with evaluation grade and admission. Conclusion: This study identified challenges in Korea's system of public reporting on $\mathrm{NH}$ quality. The accreditation system must reflect appropriate, concrete information on $\mathrm{RN}$ turnover and hospital admissions so $\mathrm{NH}$ residents are managed in a timely manner, avoiding inappropriate hospitalization.
\end{abstract}

Key Words: Nursing homes, Hospitalization, Nursing staff, Personnel turnover

\section{INTRODUCTION}

\section{Background}

South Korea is one of the fastest aging developed countries and long-term care quality is an ongoing concern for the elderly population. The Korean government introduced long-term care insurance as one form of social insurance in 2008, aiming to improve quality of life for elders and raising participation rates in family economic activity by promoting the provision of high-quality service through professional health care service [1]. In 2017, longterm care insurance beneficiaries were estimated to be about 590,000 elders, corresponding to $8.0 \%$ of all elders [1]. Long-term care insurance beneficiaries increased by $52.1 \%$; this increase was $34.9 \%$ larger than the increase in the elderly population since 2012[1]. Responding to quality concerns, public reporting of nursing home $(\mathrm{NH})$ quality has been proposed as one strategy to satisfy the diverse needs of health care settings [2].

Nursing-home-quality public reporting has been suggested as an appealing policy instrument to improve quality of care for $\mathrm{NH}$ residents [2]. In the same vein, the Korean government instituted a mandatory regulatory public-reporting system. The Korean government implemented public nursing-home-quality-rating reporting for consumers, with regular accreditation every 3 years [3]. The Korean National Health Insurance Service serves as an insurer by evaluating extension-management services for long-term care organizations including NHs, and uses evaluation results to buoy quality-of-service improvements with regular accreditation, based on Article 54

\footnotetext{
Corresponding author: Shin, Juh Hyun https://orcid.org/0000-0001-8058-7195

College of Nursing, Ewha Womans University, 52 Ewhayeodae-gil, Seodaemun-gu, Seoul 03760, Korea.

Tel: +82-2-3277-6692, Fax: +82-2-3277-2850, E-mail: juhshin@ewha.ac.kr
}

Received: Jun 9, 2020 / Revised: Jul 9, 2020 / Accepted: Aug 4, 2020

This is an open access article distributed under the terms of the Creative Commons Attribution Non-Commercial License (http://creativecommons.org/licenses/ by-nc/3.0), which permits unrestricted non-commercial use, distribution, and reproduction in any medium, provided the original work is properly cited. 
(Control and Evaluation of Long-term Care Benefits) of the Act on Long-term Care Insurance for the Elderly [3]. About $90.8 \%$ of NHs in 2015 were evaluated in administrative domains [4], environment and safety, residents' rights and responsibilities, and health care process [3]. Results of the evaluation were released to align with the public's right to know, and to increase the public's knowledge when choosing a NH [4].

To achieve appropriate standards of care, which highly relates to the quality of care, most professional care in $\mathrm{NHs}$ should be provided by registered nurse (RN) professionals because RNs are educated to deliver high-quality nursing care and help with activities of daily living for residents, based on regular assessment. In Korea, about $80 \%$ of NHs do not hire RNs because of failure to recruit RNs or to save nurse-staffing human resources by replacing $\mathrm{RNs}$ with certified nurse aides (CNAs) [5]. Despite cumulative research evidence on the importance of RNs in NHs, the number of RNs decreased about 1.6\% (from 2,683 to 2,675) in the health care workforce, whereas the number of CNAs increased by $10.18 \%$ (from 8,241 to 9,080), and care workers also increased about $6.2 \%$ (from 266,538 to 313,013 ) from 2014 in Korea [1]. The data on the turnover rate of RNs working in NHs in Korea has not been reported.

Under a clear dearth of RNs in NHs in Korea, the empirical evidence on the effects of turnover of RNs on quality-rating reporting is quite sparse. Results on residents' outcomes as a result of turnover rates has mixed reports [6]. Although no statistically significant relationship emerged to correlate RNs with hospitalization [7], very limited research reported that increased turnover of RNs related to deteriorated residents' quality-of-care outcomes in Korea $[8,9]$. In other research, turnover was not a statistically significant factor impacting residents' outcomes [10]. Despite cumulative and integrative evidence of RN staffing on $\mathrm{NH}$ residents' outcomes worldwide [11], very few studies described the effects of RN turnover on outcomes in Korea.

Understanding impact factors on mandatory government accreditation is quite challenging because many health care markets and organizational factors impact quality ratings. Very few previous researchers studied various outcomes and independent variables in the health care market. The systematic organizational climate, support or commitment, and leadership style were important factors influencing staff turnover and quality measures in NHs $[12,13]$. Large-scale NHs with more available beds used fewer antidepressants and had better quality ratings $[14,15]$. Nonprofit NHs in Korea reported better quality management, supported by a meta-analysis of 40 studies
[16]. Additional research results showed more hospitalization aligned with for-profit rather than not-for profit NHs [16].

In addition to the RN-staffing issue, it is important to investigate health care market factors, including competition among NHs or location on quality ratings, considering the unique condition of Korea. Along with the aging population, many $\mathrm{NHs}$ in Korea are deteriorating. Most $\mathrm{NHs}$ are located in specific administrative districts, whereas some districts have a very limited number of NHs [4]. Most NHs (77.4\%) are located in metropolitan and medium-sized cities, with the remainder in rural and fishing villages in Korea [17]. Consequently, competition among $\mathrm{NHs}$ varies widely by location. Location of $\mathrm{NHs}$ was an important factor influencing quality of care [18]. In Korea, $\mathrm{NHs}$ in metropolitan areas had greater improvement in quality ratings than those in rural areas [17].

A sound hospitalization framework in governmentquality-evaluation ratings is urgent in Korea. Due to additional administrative requirements to be readmitted to a $\mathrm{NH}$ after 10 days of hospitalization, family members and health care providers may be reluctant to hospitalize [1]. In Korea, even potentially avoidable hospitalizations have not yet been identified, including a lack of discussion on the benefits and drawbacks of hospitalization in Korea. RNs are more likely than other care workers to be involved in decision making on appropriate hospitalization [17]. Because the provided services for $\mathrm{NH}$ residents are quite heterogeneous compared with other health care settings, professional nurses usually decide the quality of care [18]. Greater input of RNs in NHs relates to lower rates of hospitalization [19]. Having more RNs and nurse practitioners decreases unnecessary avoidable hospitalization for $\mathrm{NH}$ residents [18].

\section{Research Purpose and Hypothesis}

The purpose of this study was to investigate the impact factor of government $\mathrm{NH}$ accreditation quality ratings in Korea. We tested a model of associations linking competition among NHs [measured by the Herfindahl-Hirschman Index (HHI); the sum of the squares of all the market shares of all facilities in a country] and location(s) (measured by geographic location based on population size), mediated by nurse turnover and residents' hospitalization on government $\mathrm{NH}$ accreditation quality ratings in Korea. We tested the hypothesized model through path analysis by evaluating the contribution of any path or combination of paths to the overall fit of this model. The specific hypotheses follow: 
$\mathrm{H} 1: \mathrm{NH}$ accreditation quality rating is influenced by $\mathrm{HHI}$

$\mathrm{H} 2$ : $\mathrm{NH}$ accreditation quality rating is influenced by location

$\mathrm{H} 3$ : $\mathrm{NH}$ accreditation quality rating is influenced by hospitalization

H4: $\mathrm{NH}$ accreditation quality rating is influenced by turnover of RN

H5: Turnover of RN is influenced by location

H6: Turnover of $\mathrm{RN}$ is influenced by $\mathrm{HHI}$

H7: Hospitalization is influenced by turnover of RN

H8: Hospitalization is influenced by HHI

\section{METHODS}

\section{Study Design}

We used a cross-sectional design to identify causal factors on $\mathrm{NH}$ quality ratings.

\section{Participants}

We collected data using disproportionately stratified cluster sampling in Korea in 2017. To gain a more representative sample of NHs across Korea, we obtained a list of all NHs from the open-access list on the Long-Term Care Insurance Service website $(\mathrm{N}=4,763)$ [19]. All NHs were stratified by bed size (less than 10 , less than 29 , more than 30 beds), location (metropolitan, medium size, and small size, or rural), randomly sampled based on the percent of NHs in each province among total NHs in Korea, using disproportionate stratified cluster sampling. Based on the distribution (\%) of NHs across 17 provinces in Korea [19], we recruited from 0 to $17 \mathrm{NHs}$ from each province. Finally, a total of $60 \mathrm{NHs}$ agreed to participate in this research and we analyzed data from $44 \mathrm{NHs}$; four NHs were excluded from the government evaluation and 12 NHs were newly opened and did not qualify for evaluation.

\section{Measurements}

\section{1) Variables.}

Institution characteristics. From 2017 June to 2017 August, we collected staffing information (RN, CNA, Care Worker numbers per $\mathrm{NH}$ ), ownership, number of beds, operation year, occupancy rate, religion of organization, hours per resident day (HPRD) of RNs, CNAs, and Care Workers as institution characteristics from administrators and nursing staff from each participating
NH. We defined HPRD as the mean hours worked by nursing staff (RN, CNA, Care Worker) divided by the total number of residents [20].

$H P R D=\frac{\text { The number of employees } \times 2080 \text { hour } / \text { year }}{\text { 365days }}$

\section{2) Independent variable.}

The two independent variables were location (metropolitan, medium size, and small size), and HHI of the health-care-market concentration. The data source for the independent variables comprised retrieval and coding of the open-access long-term care government website of the Korean National Health Insurance Service [19].

Location. We grouped the location of each NH, based on the population in that geographic area. Metropolitan area referred to places with more than a million residents; medium-sized locations have a population of about a half million million people; small-sized places have a population smaller than half a million; and rural refers to places with fewer than 50,000 people. Location information was obtained from the administrators and nursing staff in each participating $\mathrm{NH}$.

HHI. The HHI is Herfindahl-Hirschman Index a measure of the competitiveness of an industry in the market concentration of its participants [21]. To measure NH market concentration, we applied the calculation method developed by Choi et al. [14]. The data source for the total number of organizations was the open-access long-term care government website of the Korean National Health Insurance Service [19]. We divided the number of NH residents in each participating organization by the total number of organizations in each of the 17 regions and squared the total for each participating setting. We calculated the total $\mathrm{HHI}$ for all NHs in each province by summing all values in each province. The HHI ranged from 0 to 1 , with higher values indicating less competition [21].

\section{3) Mediating variables.}

Turnover rate. We determined RN turnover using the following formula, developed by the American Health Care Association [22].

Turnover rate $=\frac{\text { The number of termination at the last year }(12 \text { months })}{\text { The total number of current employees at the last year }} \times 100$

We obtained "The number of terminations in the last year (12 months)" and "The total number of current employees in the last year" from administrators and nursing 
staff from each participating $\mathrm{NH}$ and calculated the following formula based on the last year's data from the point of data collection.

Hospitalization. For NHs that agreed to participate in this research, we identified the proportion of $\mathrm{NH}$ residents admitted to hospitals for any reason in the last 3 months. Hospitalization included hospital admission due to disease, infection, or end-of-life care. We excluded admission to other NHs.

\section{4) Dependent variables.}

The Korean National Health Insurance Service [4] determines and evaluates all grades to certify residents for admission to NHs. The evaluation of each $\mathrm{NH}$ is performed by the long-term care evaluation committee consisting of gerontology experts, Korean National Health Insurance Service officials, and referees of long-term carerelated organizations. A total of three experts performs written evaluation and on-site evaluation. The team evaluates 48 indicators in five categories: operation, environment and safety, rights of $\mathrm{NH}$ residents, care process, and outcomes of care [4].

Finally, the Korean National Health Insurance Service announces NH accreditation grades. In, 2015 the NH accreditation grading (5th grade: A E) was the dependent variable; the research assistant coded data with information from the website. The $\mathrm{NH}$ evaluation and the publication about accreditation grading is performed every 3 years [4] and 2015 was the most recent outcome to match with the collected data. Based on the absolute evaluation method, A (the best) grade was assigned to NHs with a score higher than 90 of 100; B (excellent) grades exceeded a score of 80; a C (satisfactory) grade was over 70; a D (average) grade was over 60; and E (Unsatisfactory) was assigned to NHs that did not meet the mandatory requirements of the government [4]. We assigned weights to each major classification area according to the importance and difficulty of the evaluation index and determined the grade according to absolute evaluation criteria, regardless of facility size.

Control variables. We controlled personal characteristics by age and long-term care grades.

\section{Data Collection}

The project investigator and researcher recruited $\mathrm{NHs}$ to reflect the population through visits, emails, and phone calls. We sent an introductory email and set a face-to-face meeting to obtain consent forms from the administrator of each $\mathrm{NH}$. We then distributed a survey to the admin- istrators of participating organizations. The administrator answered or designated directors of nurses or social workers to fill out the survey, returned to the research team in a week, followed by two reminder emails or phone calls, if not received.

\section{Data analysis}

We analyzed all data using SPSS 23.0 and AMOS 18.0, which included frequency, percentage, mean, standard deviation, Pearson's correlation coefficient, and path analysis.

\section{Ethical consideration}

This study was approved by a university institutional review board (No. 136-4). Before collecting the data, we explained the purpose and method of this study to the participants. We explained that they could discontinue participation at any time, that their anonymity is guaranteed, and that data collection would be used only as for research purposes. In addition, we provided those who answered the questionnaire a financial reward of 100,000 won (equivalent to $\$ 100)$.

\section{RESULTS}

\section{Descriptive Statistics of Participating Organizations}

Table 1 summarizes the descriptive characteristics of participating organizations $(N=44)$. About $81.4 \%$ of organizations were nonprofit, and the average period of operation was 11.93 years. About half (47.7\%) of nursing facilities were located in metropolitan areas. Metropolitan was set as a reference variable. Small city had a low NH Accreditation-Quality Grade compared to metropolitan areas. More than half $(68.2 \%)$ of organizations were unaffiliated with religion. The average number of beds was 76.20 54 4.39. About half $(52.3 \%)$ of participating organizations received an A grade. Each organization hired 1.57 RNs on average, 1.93 CNAs, and of 27.41 care workers. The average RN HPRD was 0.17, CNA HPRD was 0.37 , and Care Worker HPRD was 3.60. The turnover rate of RNs was about $11.7 \%$, of CNAs was $10.0 \%$, and of Care Workers was $18.2 \%$. The average HHI was 0.00. Each NH had about $11.6 \%$ residents with hospitalization.

\section{Path-Analysis Results on NH Accreditation-Quality Grade}

Figure 1 shows the $\mathrm{NH}$ accreditation-quality-grade 
Table 1. Organizational Characteristics

\begin{tabular}{|c|c|c|c|c|}
\hline Variables & $\mathrm{n}(\%)$ & $\mathrm{M} \pm \mathrm{SD}$ & Minimum & Maximum \\
\hline \multicolumn{5}{|l|}{ Institution } \\
\hline $\begin{array}{l}\text { Staffing } \\
\text { RN numbers per } \mathrm{NH} \\
\text { CNA numbers per } \mathrm{NH} \\
\text { CW numbers per } \mathrm{NH}\end{array}$ & & $\begin{array}{c}1.57 \pm 3.14 \\
1.93 \pm 1.40 \\
27.41 \pm 22.08\end{array}$ & $\begin{array}{l}0 \\
1 \\
2\end{array}$ & $\begin{array}{r}17 \\
6 \\
132\end{array}$ \\
\hline $\begin{array}{l}\text { Ownership } \\
\text { Profit } \\
\text { Nonprofit }\end{array}$ & $\begin{array}{r}8(18.6) \\
36(81.4)\end{array}$ & & & \\
\hline Number of beds & & $76.20 \pm 54.39$ & 9 & 296 \\
\hline Operation year & & $11.93 \pm 6.99$ & 2 & 34 \\
\hline HHI & & $0.000559 \pm 0.000857$ & 0.000025 & 0.003761 \\
\hline Occupancy rate & (91.3) & & & \\
\hline Hospitalization rate & (11.6) & & & \\
\hline $\begin{array}{l}\text { Location } \\
\text { Metropolitan (over a million) (reference) } \\
\text { Medium size (around a half million } \sim \text { million) } \\
\text { Small size (5 around a half million) } \\
\text { Rural (below } 50 \text { thousand) }\end{array}$ & $\begin{aligned} & 21(47.7) \\
& 7(15.9) \\
& 12(27.3) \\
& 4(9.1)\end{aligned}$ & & & \\
\hline $\begin{array}{l}\text { Religion of organization } \\
\text { Protestant } \\
\text { Catholic } \\
\text { Buddhist } \\
\text { None }\end{array}$ & $\begin{array}{c}9(20.5) \\
2(4.5) \\
3(6.8) \\
30(68.2)\end{array}$ & & & \\
\hline $\begin{array}{l}2015 \text { Facility evaluation by the KNIS } \\
\text { A (the very best) } \\
\text { B (excellent) } \\
\text { C (satisfactory) } \\
\text { D (average) } \\
\text { E (unsatisfactory) }\end{array}$ & $\begin{array}{r}23(52.3) \\
8(18.2) \\
6(13.6) \\
7(15.9) \\
0(0.0)\end{array}$ & & & \\
\hline $\begin{array}{l}\text { HPRD } \\
\text { RN } \\
\text { CNA } \\
\text { Care worker }\end{array}$ & & $\begin{array}{l}0.17 \pm 0.19 \\
0.37 \pm 0.24 \\
3.60 \pm 1.41\end{array}$ & $\begin{array}{l}0.00 \\
0.00 \\
1.35\end{array}$ & $\begin{array}{l}0.63 \\
1.14 \\
8.71\end{array}$ \\
\hline $\begin{array}{l}\text { Turnover rate } \\
\text { RN } \\
\text { CNA } \\
\text { Care worker }\end{array}$ & $\begin{array}{l}(11.7) \\
(10.0) \\
(18.2)\end{array}$ & $\begin{array}{r}11.68 \pm 24.00 \\
9.95 \pm 19.84 \\
18.19 \pm 17.38\end{array}$ & $\begin{array}{l}0 \\
0 \\
0\end{array}$ & $\begin{array}{r}100 \\
60 \\
86\end{array}$ \\
\hline
\end{tabular}

$\mathrm{CNA}=$ Certified nurse aide; $\mathrm{CW}=$ Care worker; $\mathrm{HHI}=$ Herfindahl-hirschman index; $\mathrm{HPRD}=$ Hours per resident day; KNIS=Korean National Insurance Service; $\mathrm{NH}=\mathrm{Nursing}$ home; $\mathrm{RN}=$ Registered nurse.

model tested. We conducted path analysis using SPSS 23.0. Path analysis may be applied as a substitute for regression to examine mediating factors in a model and cause-and-effect relationships among variables [23]. Path analysis is a useful statistical method to test causal relationship in a cross-sectional dataset [24]. In this study, squared multiple correlation of turnover_RN was .17, hospitalization was .15 , NH quality rating was .31 .

\section{Model Fit}

For the original model, we expected all hypothesized paths would impact public reporting of NH quality. Applying model trimming in path analysis, we deleted some insignificant pathways and added significant correlation pathways while authorizing the model fit [25]. In the level of nurse staffing level, the turnover of CNAs and Care 


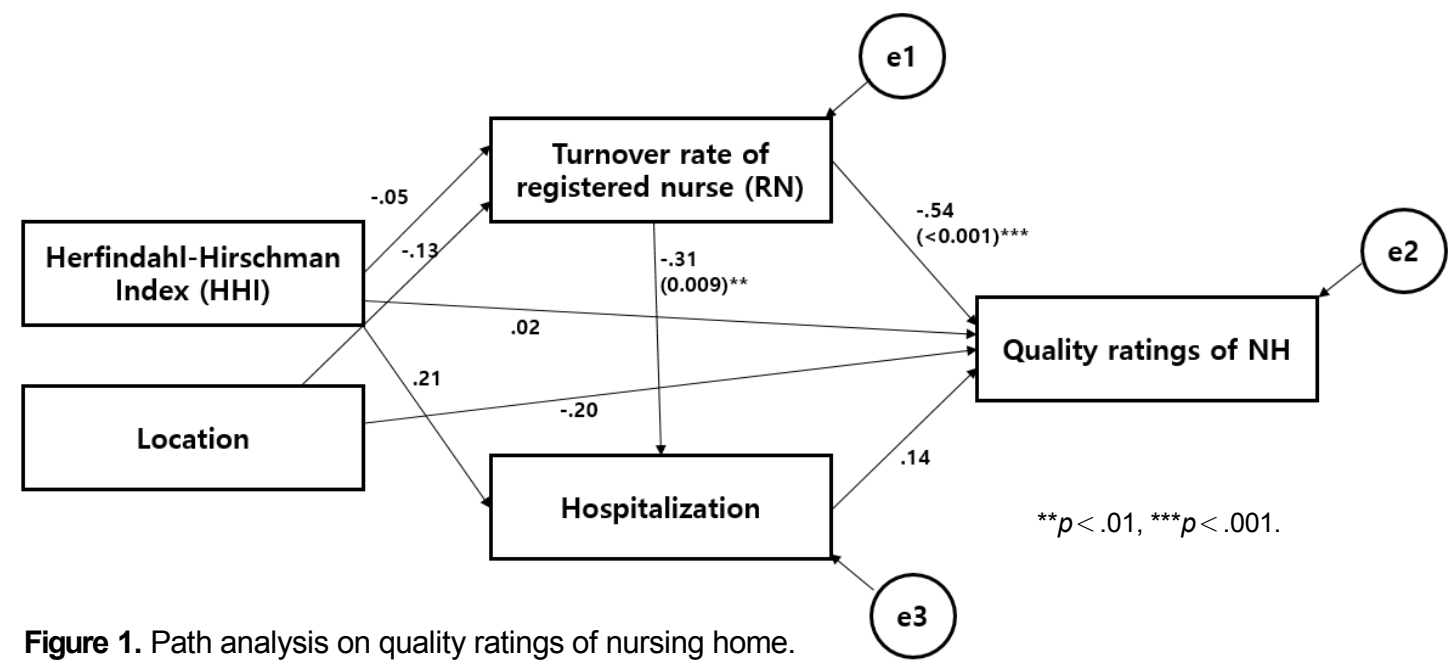

Table 2. Model Fit

\begin{tabular}{lcc}
\hline Indices & Acceptable value & $\begin{array}{c}\text { Fitness indices of } \\
\text { hypothetical model }\end{array}$ \\
\hline NPAR & 19 \\
DF & 1 \\
CMIN & $>.100$ & .781 \\
TLI & $>.900$ & 1.179 \\
CFI & $<.050$ & 1.000 \\
RMSEA & .000 \\
\hline
\end{tabular}

$\mathrm{CFI}=$ Comparative fit index; $\mathrm{CMIN}=x^{2}$ minimum discrepancy; $\mathrm{DF}=$ Degrees of freedom; NPAR $=$ Number of distinct parameters to be estimated; RMSEA=Root mean square residual; TLI=Tucker-lewis index.

Workers was deleted; for the organizational levels, the size, profit or not-for profit organizations, distribution of functional severity of residents, the presence of administrators with RN license, and religious basis of the organizations were deleted. Figure 1 reports parameter estimates for the final model. We used the SPSS 23.0 structural-equation-modeling statistical package. We evaluated the degree of consistency between actual collected data and the hypothesized model with some model-fit measures (Table 2). Model fit emerged as follows: Root-meansquare error of approximation was 0 , and the divergence per degree of freedom was adequate because it was less than .05 [26]. The comparative fit index was 1.00, which was quite acceptable because .90 indicates a more perfect fit [26]. The Tucker-Lewis Index was acceptable because it was nearly 1 . The goodness-of-fit index was 1 [26].

Path coefficients in the tested model appear in Table 3. Two path coefficients were significant at .001. The high turnover of professional RNs related to deteriorated gov- ernment nursing-home-quality ratings, and more hospitalizations related to better nursing-home-quality ratings. $\mathrm{RN}$ turnover related negatively to hospitalizations and $\mathrm{NH}$ accreditation-quality ratings. $\mathrm{RN}$ turnover was statistically significant, sporting an evaluation grade with a path loading of $\beta$ (standardized coefficient) $=-.54$. That is, increasing turnover of RNs aligned with decreased accreditation-quality rating. $\mathrm{RN}$ turnover was statistically significant with hospitalizations: $\beta=-.31$; that is, increasing $\mathrm{RN}$ turnover aligned with decreased hospitalization of $\mathrm{NH}$ residents.

\section{DISCUSSION}

This study established the most comprehensive examination of related factors on public reporting of $\mathrm{NH}$ quality in Korea. The relationship of competition among NHs and location, mediated by nurse turnover and hospitalization with the public reporting of the nursing-homequality rating is an important topic for $\mathrm{NH}$ administrators, health care policymakers, researchers, and nursing educators. The model proposed in this study builds on the proposition that the level of RN turnover and hospitalization impacts government-led $\mathrm{NH}$ accreditation-quality evaluation grades. From this viewpoint, the turnover rate of RNs was a significant factor in nursing-homequality ratings and hospitalizations of $\mathrm{NH}$ residents.

In this study, we found no statistically significant relationship between the location and the competitive index of NHs on accreditation evaluation grade aligned with turnover of RNs and hospitalization of $\mathrm{NH}$ residents in the path analysis. These findings are consistent with a previous study in that the nature of rural NHs did not relate to avoidable hospitalization [19]. Also, higher turn- 
Table 3. Regression Weights

\begin{tabular}{lllcrrrrr}
\hline & Items & & $\begin{array}{c}\text { Standardized } \\
\text { coefficients }\end{array}$ & $\begin{array}{c}\text { Unstandardized } \\
\text { coefficients }\end{array}$ & SE & CR & $p$ & SMC \\
\hline Turnover_RN & $\leftarrow$ & Location & -.13 & $-6,296.19$ & $6,344.03$ & -0.99 & .321 & .17 \\
Turnover_RN & $\longleftarrow$ & HHI & -.05 & $-2,966,145.44$ & $7,555,363.84$ & -0.39 & .695 \\
Hospitalization & $\leftarrow$ & Turnover_RN & -.31 & 0.00 & 0.00 & -2.60 & .009 & .15 \\
Hospitalization & $\leftarrow$ & HHI & .21 & 468.67 & 269.41 & 1.74 & .082 \\
NH quality rating & $\leftarrow$ & HHI & .02 & 30.19 & 167.23 & 0.18 & .857 & .31 \\
NH quality rating & $\leftarrow$ & Location & -.20 & -0.23 & 0.14 & -1.66 & .098 \\
NH quality rating & $\leftarrow$ & Hospitalization & .14 & 0.08 & 0.08 & 1.06 & .291 \\
NH quality rating & $\leftarrow$ & Turnover_RN & -.54 & 0.00 & 0.00 & -4.23 & $<.001$ \\
\hline
\end{tabular}

$\mathrm{CR}=$ Critical ratio; $\mathrm{HHI}=$ Herfindahl-hirschman index; $\mathrm{NH}=$ Nursing home; $\mathrm{RN}=$ Registered nurse; $\mathrm{SE}=$ Standardized estimates; $\mathrm{SMC}=\mathrm{Squared}$ multiple correlation.

over of RNs related to increased hospitalization [18]. It seems that health-care-market factors other than competition among $\mathrm{NHs}$ or location may impact $\mathrm{NH}$ government-accreditation-evaluation grade. Possible factors may include subsidy by the government; the process of nursing care; the mission, environment, and perspectives of care among health care professionals; and perceived competition by staff, culture, or NH residents' characteristics. Further research should be conducted to consider these factors.

The mediating factors in this study were turnover of RNs and hospitalizations from NHs. Until now, mixed results persist about the effects of public reporting of $\mathrm{NH}$ quality using NH Compare website information from the Centers for Medicare \& Medicaid Services, initiated in 2002. Despite improvements, postacute-care-quality items [3] had a very marginal impact on customers' market share [3]. However, no previous studies considered the turnover of RNs and hospitalization.

The turnover rate of RNs in this study is reported as $11.7 \%$; very low compared with that of the United States (47\%) [26]. This study also reported that the high turnover in RN staffing results in fewer hospitalizations. However, considering the specific staffing conditions in Korea-that CNAs can replace RNs, and visiting doctors only visit NHs once per month-having well-timed medical and nursing care in hospitals is very important to avoid prompt deterioration of health-related problems like pneumonia or infections of NH residents [17]. The interpretation should be performed cautiously for hospitalization. Previous researchers suggested that a stable supply of RNs in $\mathrm{NHs}$ is a critical factor in $\mathrm{NH}$ management, including timely and appropriate hospitalization [9].
Stable RN staffing can be an important political moderator of $\mathrm{NH}$ outcomes. High turnover may make it difficult for RNs to have a familiar relationship with $\mathrm{NH}$ residents, consistent supervision of unlicensed nursing staff, or the ability to assess $\mathrm{NH}$ residents appropriately [15]. Regulations on a hospital length of stay less than 10 days to be eligible for a NH stay influences hospitalizations in Korea [1]. Thus, to resolve inappropriate or untimely hospitalization from NHs in Korea, political change should follow. One solution may be benchmarking, as in the U.S. system: Medicaid bed-hold policies mean NH residents may be readmitted to NHs after acute hospital care, due to state subsidies [27]. Furthermore, most Koreans and health care professionals in Korea do not have government data on appropriate or avoidable hospitalizations, so it is of timely importance to conduct further research on nurse staffing and hospitalization. Furthermore, data on hospitalization in this study did not consider the possibilities of preventable hospitalization and timely hospitalizations. Future public reporting of $\mathrm{NH}$ quality should consider not only the number of RNs but also their turnover rate.

$\mathrm{NH}$ residents may transfer to hospitals for acute care or hospice care. Timely appropriate care in hospitals or unavoidable hospitalizations remain a concern of care. During the transition from $\mathrm{NH}$ to hospital, $\mathrm{NH}$ residents may experience delirium, infectious diseases, functional deterioration, anorexia, falls, incontinence, inappropriate tube feeding, unexpected medical treatment, and end-oflife care [28]. Inappropriate hospitalization transfers from $\mathrm{NHs}$ due to improper care increase unnecessary health care costs (about $\$ 2.7$ billion yearly) [28]. Some comparison studies reported that the quality of treatment out- 
comes was not different between NHs and hospital care [29]. About $25 \sim 46 \%$ of $\mathrm{NH}$ residents were hospitalized for end-of-life care in the United States [30], but appropriate end-of-life care may be threatened because of hospitalization. Consistent, stable, and professional care by RNs who are familiar with the health care needs of $\mathrm{NH}$ residents should be guaranteed. The hospitalization of $\mathrm{NH}$ residents should be addressed systematically and continuously; RNs are responsible for decisions about the necessity for patients to receive intensive care at hospitals, about follow-up after transitioning to hospitals, and about readmission to $\mathrm{NHs}$ after patients receive medical care at hospitals. Furthermore, scientific assessment and monitoring of NH residents' weight or intake of food or beverage, and symptoms or signs of disease may reduce needless hospitalization for residents, consistent with previous research [28]. No researchers described the hospitalization of NH residents in NHs. Further research should investigate related factors on hospitalization aligned with quality measures.

The United States has been considered a pioneer in measuring quality outcomes in long-term care settings, and Korea needs to use the U.S. quality-control system as a benchmark. On the U.S. nursing-home-compare website (which corresponds to the National Long Term Care Insurance website, in Korea), the percentage of $\mathrm{NH}$ residents compared to hospitalizations or emergency visits is considered essential information for consumers because these indicators show when more $\mathrm{NH}$ residents are admitted to hospitals because health care providers may not have assessed or appropriately cared for them [9].

This study contributes to nursing practice, education, and research. In NH practice, systematic efforts to reduce the turnover rate of RNs should proceed to lower the hospitalization rate of residents. $\mathrm{NH}$ administrators and $\mathrm{RNs}$ should also consider competition among NHs and location for better quality control, which relates to public reporting. In nursing education, it is necessary to educate RNs and nursing students about factors related to quality ratings for NHs. More research is needed to support programs that decrease the turnover of $\mathrm{RNs}$ in $\mathrm{NHs}$ and thereby increase the quality rating of NHs.

This study has several limitations. First, external validity was threatened by the small number of participating NHs. Therefore, results should be carefully considered. Follow-up studies are needed with similar data collection. Second, power analysis was not feasible in this study due to a lack of available software. Participating organizations in this study were $44 \mathrm{NHs}$ and were the unit of analysis. The 44 organizations have a total of 3,980 resi- dents. However, the unit of analysis was "NH." Power analysis on individual parameters and the total model differ. Future researchers may apply Monte Carlo or Satorra and Saris for power calculation. Third, only a few of the various variables that could affect $\mathrm{NH}$ Accreditation-Quality Grades were used in this study. Future study on other factors affecting the quality ratings of $\mathrm{NHs}$ also need to be addressed.

\section{CONCLUSION}

In conclusion, this study is the first to examine the broader impact of competition among NHs and location, mediated by nurse turnover and residents' hospitalization on quality ratings for $\mathrm{NH}$ public reporting in Korea. This study identified challenges in Korea's system of public reporting on $\mathrm{NH}$ quality. The accreditation system should reflect appropriate, concrete information on turnover of RNs and hospitalization so residents in NHs are managed in a timely manner, avoiding inappropriate hospitalization.

\section{CONFLICTS OF INTEREST}

The authors declared no conflict of interest.

\section{AUTHORSHIP}

Study conception and design acquisition - SJH and SI-S; Data collection - SJH and LJY; Analysis and interpretation of the data SJH and SI-S; Drafting and critical revision of the manuscript SJH and LJY; Final approval - SJH, SI-S, and LJY.

\section{ACKNOWLEDGEMENT}

This work was supported by the National Research Foundation of Korea (NRF) grant funded by the Korea government (Ministry of Science, ICT \& Future Planning (2017R1A2B4003282).

\section{REFERENCES}

1. National Health Insurance Service. Long term care insurance statistical yearbook 2018 [Internet]. Wonju: National Health Insurance Service; 2018 [updated 2018 Jan 2, cited 2018 Apr 20]. Available from:

http://www.khiss.go.kr/board/view?pageNum=1\&rowCnt =10\&no1=260\&linkId=175347\&menuId=MENU00309\&schT ype $=0 \&$ schText $=\&$ boardStyle $=\&$ categoryId $=\&$ continent $=\& \mathrm{~s}$ chStartChar $=\&$ schEndChar $=\&$ country $=$.

2. Werner RM, Norton EC, Konetzka RT, Polsky D. Do consumers respond to publicly reported quality information? evidence from nursing homes. Journal of Health Economics. 
2012;31(1):50-61.

https://doi.org/10.1016/j.jhealeco.2012.01.001

3. National Health Insurance Service. Regular evaluation plans for long-term care institutions 2018 [Internet]. Wonju: National Health Insurance Service; 2018 [updated 2018 Jan 19; cited 2019 Jan 15]. Available from

http://www.longtermcare.or.kr/npbs/d/m/000/moveBoard View?menuId=npe0000000770\&bKey=B0009\&search_board $\mathrm{Id}=60221$.

4. National Health Insurance Service. Long-term care institution (facility benefit) evaluation result 2016 [Internet]. Wonju: National Health Insurance Service; 2016 [updated 2016 Apr 22; cited 2019 Jan 15]. Available from

http://www.longtermcare.or.kr/npbs/d/m/000/moveBoard View?menuId=npe0000000770\&bKey=B0009\&search_board $\mathrm{Id}=60016$.

5. Lee JM, Park MJ, Han EJ, Suh EE. A study on management of medical needs of the elderly in Korean long-term care facilities 2012-19. Seoul: Health Insurance Policy Institute, 2012. $202 \mathrm{p}$.

6. Castle NG, Anderson RA. Caregiver staffing in nursing homes and their influence on quality of care: using dynamic panel estimation methods. Medical Care. 2011;49(6):545-52. https://doi.org/10.1097/MLR.0b013e31820fbca9

7. Intrator O, Castle NG, Mor V. Facility characteristics associated with hospitalization of nursing home residents: results of a national study. Medical Care. 1999:37(3):228-37. https://doi.org/10.1097/00005650-199903000-00003

8. Shin JH. Why do we require registered nurses in nursing homes? using longitudinal hierarchical linear modeling. Journal of Nursing Scholarship. 2018;50(6):705-13. https://doi.org/10.1111/jnu.12412

9. Shin JH, Hyun TK. Nurse staffing and quality of care of nursing home residents in Korea. Journal of Nursing Scholarship. 2015 47(6):555-64. https://doi.org/10.1111/jnu.12166

10. Shin JH, Shin IS. The effect of registered nurse on nursing home residents' outcomes, controlling for organizational and health care market factors. Geriatric Nursing. 2019;40(3):296-301. https:// doi.org/10.1016/j.gerinurse.2018.11.004

11. Dellefield ME, Castle NG, McGilton KS, Spilsbury K. The relationship between registered nurses and nursing home quality: an integrative review (2008-2014). Nursing Economics. 2015;33 (2):95-108.

12. Backhaus R, Rossum EV, Verbeek H, Halfens RJ, Tan FE, Capezuti E, et al. Work environment characteristics associated with quality of care in Dutch nursing homes: a cross-sectional study. International Journal of Nursing Studies. 2017;66:15-22. https://doi.org/10.1016/j.ijnurstu.2016.12.001

13. Castle NG, Decker FH. Top management leadership style and quality of care in nursing homes. The Gerontologist. 2011;51
(5):630-42. https://doi.org/10.1093/geront/gnr064

14. Choi JS, Choi S, Lee S, Han E, Kim M. Does market competition facilitate resident-centred care among nursing homes? a comparative analysis. Asia Pacific Journal of Social Work and Development. 2016;26(1):15-28.

https://doi.org/10.1080/02185385.2016.1150874

15. Lapane KL, Hughes CM. Which organizational characteristics are associated with increased management of depression using antidepressants in US nursing homes? Medical Care. 2004;42 (10):992-1000.

https://doi.org/10.1097/00005650-200410000-00008

16. Comondore VR, Devereaux PJ, Zhou Q, Stone SB, Busse JW, Ravindran NC, et al. Quality of care in for-profit and not-forprofit nursing homes: systematic review and meta-analysis. BMJ. 2009;339:b2732. https://doi.org/10.1136/bmj.b2732

17. Chin Y, Choi K. The related factors with improvement of longterm care need of residents and quality of service in long-term care facility. The Korean Journal of Health Service Management. 2014;8(1):51-64. https://doi.org/10.12811/kshsm.2014.8.1.051

18. de Oliveira WIF, Hernández PJS, de Meneses Sousa K, da Silva G, da Silva Gama ZA. Quality of care in nursing homes in Brazil. Journal of the American Medical Directors Association. 2017;18(7):636.e13-9.

https://doi.org/10.1016/j.jamda.2017.04.007

19. National Health Insurance Service. Long-term care insurance statistics yearbook 2018 [Internet]. Wonju: National Health Insurance Service; 2019 [updated 2019 Jan 3; cited 2019 Jan 16]. Available from:

http://www.khiss.go.kr/board/view?pageNum=1\&rowCnt =10\&no1=260\&linkId=175347\&menuId=MENU00309\&sch Type $=0 \&$ schText $=\&$ boardStyle $=\&$ categoryId $=\&$ continent $=\&$ schStartChar=\&schEndChar $=\&$ country $=$

20. Pennsylvania department of health. Guidance for calculating direct nursing care hours [Internet]. Harrisburg, PA: Pennsylvania Department of Health; 2016 [updated 2016 Dec 9; cited 2018 Jun 11]. Available from:

http://www.padona.com/cms/uploads/attachment/Provi der_Pointers_on_Calculating_Nursing_Hours_rev_Nov_2016 .pdf

21. Susilo YO, Axhausen KW. Repetitions in individual daily activity-travel-location patterns: a study using the HerfindahlHirschman Index. Transportation. 2014;41:995-1011. https://doi.org/10.1007/s11116-014-9519-4

22. American Health Care Association. Staffing survey report [Internet]. Washington, DC: American Health Care Association; 2011 [updated 2012 Apr 11; cited 2018 Jun 11]. Available from: http://www.ahcancal.org/research_data/staffing/Docume nts/2011\%20Staffing\%20Survey\%20Report.pdf

23. Baron RM, Kenny DA. The moderator-mediator variable dis- 
tinction in social psychological research: conceptual, strategic, and statistical considerations. Journal of Personality and Social Psychology. 1986;51(6):1173-82.

https://doi.org/10.1037//0022-3514.51.6.1173

24. Furuta M, Komiya-Nonaka M, Akifusa S, Shimazaki Y, Adachi M, Kinoshita T, et al. Interrelationship of oral health status, swallowing function, nutritional status, and cognitive ability with activities of daily living in Japanese elderly people receiving home care services due to physical disabilities. Community Dentistry and Oral Epidemiology. 2013;41(2):173-81. https://doi.org/10.1111/cdoe.12000

25. Duncan OD. Path analysis: sociological examples. American Journal of Sociology. 1996;72(1):1-16.

https://doi.org/10.1086/224256

26. Hooper D, Coughlan J, Mullen MR. Structural equation modelling: guidelines for determining model fit. Electronic Journal of Business Research Methods. 2008;16(1):53-60.
https://doi.org/10.21427/D7CF7R

27. Unruh MA, Grabowski DC, Trivedi AN, Mor V. Medicaid bed-hold policies and hospitalization of long-stay nursing home residents. Health Services Research. 2013;48(5):1617-33. https://doi.org/10.1111/1475-6773.12054

28. Charette SL. Hospitalization of the nursing home patient. Journal of the American Medical Directors Association. 2003;4(2): 90-4. https://doi.org/10.1016/S1525-8610(04)70281-9

29. Givens JL, Selby K, Goldfeld KS, Mitchell SL. Hospital transfers of nursing home residents with advanced dementia. Journal of the American Geriatrics Society. 2012;60(5):905-9. https://doi.org/10.1111/j.1532-5415.2012.03919.x

30. Menec VH, Nowicki S, Blandford A, Veselyuk D. Hospitalizations at the end of life among long-term care residents. The Journals of Gerontology, Series A: Biomedical Sciences and Medical Sciences. 2009;64A(3):395-402.

https://doi.org/10.1093/gerona/gln034 